Article

\title{
Identification and Validation of Quantitative Trait Loci for Grain Number in Rice (Oryza sativa L.)
}

\author{
Xiaojun Niu ${ }^{1,2}$, Yujun Zhu ${ }^{1}$, Zhichao Sun ${ }^{1}$, Sibin $\mathrm{Yu}^{2}{ }^{2}$, Jieyun Zhuang ${ }^{1}{ }^{\mathbb{C}}$ and Yeyang Fan ${ }^{1, *}$ \\ 1 State Key Laboratory of Rice Biology and Chinese National Center for Rice Improvement, China National \\ Rice Research Institute, Hangzhou 310006, China; xiaojunwords@126.com (X.N.); yjzhu2013@163.com (Y.Z.); \\ sunzhichao109962@163.com (Z.S.); zhuangjieyun@caas.cn (J.Z.) \\ 2 State Key Laboratory of Crop Genetic Improvement and National Center of Plant Gene Research (Wuhan), \\ Huazhong Agricultural University, Wuhan 430070, Hubei, China; ysb@mail.hzau.edu.cn \\ * Correspondence: fanyeyang@caas.cn; Tel.: +86-571-6337-0364
}

Received: 19 December 2019; Accepted: 20 January 2020; Published: 27 January 2020

check for updates

\begin{abstract}
Grains number is one of the most important agronomic traits in the determination of rice productivity. To explore the underlying genetic basis of grain number in rice, quantitative trait locus (QTL) analysis was performed using three recombinant inbred line populations derived from indica rice crosses of Teqing/IRBB lines, Zhenshan 97/Milyang 46, and Xieqingzao/Milyang 46, respectively. A total of 58 QTLs distributed on all 12 rice chromosomes were identified, including 22 for number of grains per panicle (NGP), 17 for number of spikelets per panicle, and 19 for spikelet fertility. The individual QTL counted for 1.5 to $22.1 \%$ of phenotypic variation. Among them, 15 QTLs shared by two or three populations and eight QTLs showed large effects with $R^{2}$ larger than $10 \%$. Furthermore, three QTLs with minor effects for NGP, qNGP5.5, qNGP9.1, and qNGP12.1, were detected and validated by eliminating the segregation of major-effect QTL using four residual heterozygote-derived populations. These results not only enrich our understanding of the mechanism of grain number, but also provide a foundation for cloning and selecting candidate for marker-assisted selection breeding in rice.
\end{abstract}

Keywords: rice; grain number; QTL; minor effect; residual heterozygote

\section{Introduction}

Rice (Oryza sativa L.) is one of the most important cereal crops in the world, playing a key role in meeting the demand of food for a growing global population. During the last 60 years, grain yield has progressively increased in Yangtze River basin, the main indica rice-producing area in China, and such an increase is mainly attributed to the expanded sink size as a result of more number of spikelets per panicle (NSP), especially for the case of super rice [1]. Yang et al. analyzed the data of national rice regional trial in southern China from 1986 to 2002 and found that number of grains per panicle (NGP) played an important role in improving both the yield and rice quality based on suited number of effective panicles and grain weight [2]. Therefore, it is essential to understand the molecular mechanism of grain number in rice.

All three yield components-panicle number, grain number, and grain weight-are typical quantitative traits that are controlled by polygenes referred to as quantitative trait loci (QTLs) [3]. QTL mapping is an efficient strategy to dissect the molecular mechanisms of rice yield traits such as NGP [4]. Along with molecular marker technology development, hundreds of QTLs distributed on rice 12 chromosomes had been identified for NGP and NSP (www.gramene.org/archive/QTL data). Most of them were detected in different mapping populations such as $\mathrm{F}_{2}$, doubled haploid lines, and recombinant inbred lines (RILs) [5-10]. Several dozens of QTLs for NGP and NSP have 
been fine-mapped [11-17]. So far, eight QTLs have been cloned. Gn1a and NOG1 regulate grain numbers [18,19]. DEP1, IPA1/WFP, APO1, and GNP1 control panicle architecture and meristems [20-25]. NAL1 mainly controls panicle size and plant architecture [26,27]. Ghd7 presents large pleiotropic effect on NGP, heading date and plant height $[28,29]$. These findings have greatly promoted the dissection of genetic bases of rice grain number.

However, these studies mainly focused on major-effect QTLs, few studies investigated minor-effect QTLs. Recently, studies have shown that QTLs with minor effect also play a role in the regulation of important agronomic traits in rice [30]. Hence, identification of minor-effect QTLs would enrich our knowledge of genetic and molecular network regulating rice grain number. Usually, minor-effect QTLs are more sensitive to genetic background and environment than major-effect QTLs [31,32]. Identification of minor-effect QTLs would be facilitated by eliminating the effect of major-effect QTLs. The use of residual heterozygote (RH) to construct near-isogenic lines (NILs) or secondary segregation populations is an effective strategy for identification and verification of minor-effect QTLs [33,34]. Based on this approach, several QTLs for yield-related trait have been fine-mapped [35,36].

In the present study, QTL analysis for NGP, NSP, and spikelet fertility (SF) was performed using three RIL populations. Three minor-effect QTLs on chromosome 5, 9 and 12 were identified using one RH-derived population fixing the major-effect QTLs and further validated in the new secondary segregation population under more homozygous genetic background, respectively.

\section{Materials and Methods}

\subsection{Plant Materials}

A total of seven populations developed from crossing of indica rice were used. For primary mapping, the previously developed three RIL populations were already used to analyze grain quality and yield-related traits, including Teqing/IRBB lines (TI), Zhenshan 97/Milyang 46 (ZM) and Xieqingzao/Milyang $46(\mathrm{XM})$, respectively [10,37-41]. Teqing, Zhenshan 97 and Xieqingzao are female parents, while IRBB lines and Milyang 46 are male parents. All of the parental lines have been widely used in commercial breeding and production of three-line hybrid rice in China, among which Zhenshan 97 and Xieqingzao are maintainer lines and used as early-season varieties, others are restorer lines and used as middle-season rice varieties in the middle-lower reaches of Yangtze River basin. For TI, phenotypic data of 203 lines in 2009 and 2010 and genetic maps with 127 markers, spanning 1198 cM were used to identify QTLs for yield heterosis [38]. In this study, extra two years' phenotypic data were added, and genetic linkage map had been updated to comprise 135 markers spanning 1345 cM [42]. For ZM, phenotypic data of 243 lines in 1999 and 2000 and genetic map with 158 makers, spanning $1288 \mathrm{cM}$ were used to detect QTLs for yield traits [10]. In this work, phenotypic data for two more years were added. Genetic linkage map had been updated to consist of 256 markers and span $1815 \mathrm{cM}$ [39]. For XM, phenotypic data of 209 lines was not used in any QTL mapping for NGP, NSP, and SF. A genetic map consisting of 240 markers and spanning $2080 \mathrm{cM}$ [39], and phenotypic data of three years were used in this study.

For further mapping and validation, four RH-derived populations were used, and they were derived from one RH of the cross TQ/IRBB52 as described below and illustrated in Figure 1. One $\mathrm{F}_{7}$ plant carrying 13 heterozygous segments distributed on 10 chromosomes was selected and selfed to produce $S_{1}$ population consisting of 251 individuals, which was named Ti52-2. QTLs were determined from data generated from the $\mathrm{S}_{1: 2}$ and $\mathrm{S}_{1: 4}$ families of Ti52-2. QTLs for heading date had been detected using Ti52-2 in previous study [42].

Three RHs were selected from Ti52-2 covering qNGP5.5, qNGP9.1 and qNGP12.1, respectively. The first one carried three heterozygous segments, which were RM18927-RM274 on chromosome 5, RM20731 on chromosome 6 and pTA248-RM5926 on chromosome 11. The second one included four heterozygous segments, which were RM12210 on chromosome 1, RM23662-RM1896 on chromosome 9, RM6704-RM7300 on chromosome 10 and RM1233-RM5926 on chromosome 11. The last one consisted 
of two heterozygous segments, which were RM16252-RM335 on chromosome 4 and RM3246-RM511 on chromosome 12. They were selfed to produce three $S_{1}$ populations designated ZC5, ZC9 and ZC12 consisting of 216, 203 and 241 plants, respectively.

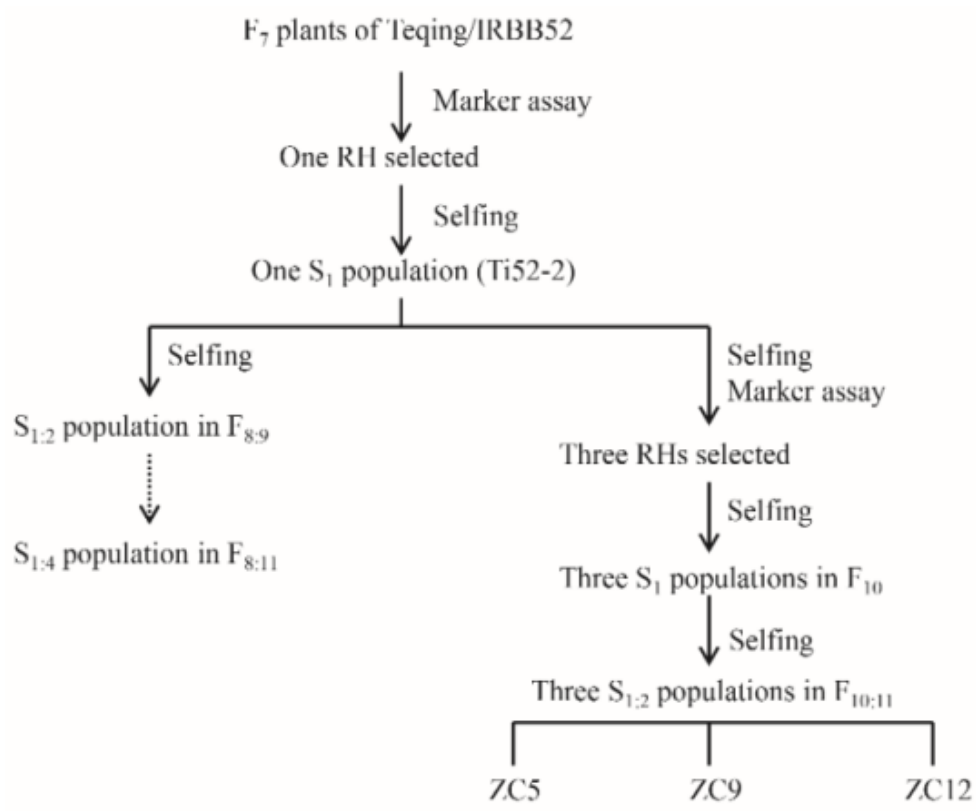

Figure 1. Construction of the rice populations used in this study. $\mathrm{RH}$, residual heterozygote.

\subsection{Field Trials and Phenotypic Evaluation}

All the populations were grown from May to September in the paddy fields at the China National Rice Research Institute in Hangzhou, Zhejiang, China. A randomized complete block designed with two replications was used for all trials. In each replication, one line was grown as a single row of 12 plants. The planting density was $16.6 \mathrm{~cm} \times 26.7 \mathrm{~cm}$ in all trials. Field management followed local agricultural practice. At maturity, five middle plants of each line were harvested in bulk for three RIL populations, and ten middle plants of each line for other populations. NGP, NSP, and SF were measured in three RIL populations and Ti52-2, whereas only NGP was measured in ZC5, ZC9 and ZC12 populations.

\subsection{DNA Marker Analysis}

Marker data and the linkage map have been available except for ZC5 [39,42]. For ZC5, A total of eight polymorphic DNA markers were employed, including three on chromosome 5, one on chromosome 6 and four on chromosome 11, respectively (Table S1). DNA was extracted using $2 \mathrm{~cm}$ long-leaf samples collected from the middle 10 plants of each line following the method of Zheng et al. [43]. PCR amplification followed the method of Chen et al. [44]. The products were visualized on $6 \%$ or $8 \%$ non-denaturing polyacrylamide gels by silver staining for seven simple sequence repeat markers, and $2 \%$ agarose gels using GelRed (Biotium, Fremont, CA, USA) staining for one sequence-tagged site marker. Linkage map was constructed using Mapmaker/Exp 3.0 (Whitehead Institute for Biomedical Research, Cambridge, MA, USA) [45], Kosambi function was used to calculate the genetic distance.

\subsection{Data Analysis}

Phenotypic values of two replications were averaged for each line and used for data analysis in each trial. Basic descriptive statistics, including mean value, standard deviation, coefficient of variation, the minimum and maximum trait values, skewness, and kurtosis were computed in each trial. The mean value of each trait was used to calculate the correlation coefficients between each trait. 
For three RIL and Ti52-2 populations, QTLs were determined using the MET (multi-environment trials) functionality in QTL IciMapping V4.1 software (Chinese Academy of Agricultural Sciences, Beijing, China), taking different years as different environments [46]. LOD thresholds were calculated with 1000 permutation tests $(p<0.05)$ for each trait and used to declare a putative QTL, which were 4.3-4.8, 4.3-4.8, and 4.4-4.6 for NGP, NSP, and SF in three RIL populations, as well as 2.8, 2.7 and 2.8 in Ti52-2, respectively. QTLs detected were designated following the rule proposed by McCouch and CGSNL [47].

For ZC5, ZC9 and ZC12 populations, QTLs were determined using CIM (composite interval mapping) approach in Windows QTL Cartographer 2.5 (North Carolina State University, Raleigh, NC, USA) [48]. A threshold of $L O D>2.0$ was used for claiming a putative QTL.

\section{Results}

\subsection{Phenotypic Variance}

Descriptive statistics of the NGP, NSP, and SF in each trial of three RIL populations were presented in Table 1. The three traits were continuously distributed with low skewness and kurtosis, showing a typical pattern of quantitative variation. Generally, the mean values for all three traits were higher in TI than in ZM and XM. In terms of phenotypic differences between the female and male parents, significant differences $(p<0.05)$ were observed for NGP and NSP only in TI. The female parent TQ had higher values of NGP and NSP compared with the male parent.

Table 1. Phenotypic performance of number of grains per panicle (NGP), number of spikelets per panicle (NSP) and spikelet fertility (SF) in the three recombinant inbred line (RIL) populations.

\begin{tabular}{|c|c|c|c|c|c|c|c|c|c|c|}
\hline \multirow[t]{2}{*}{ Trait $^{a}$} & \multirow[t]{2}{*}{ Population $^{b}$} & \multirow[t]{2}{*}{ Year } & \multirow[t]{2}{*}{ Mean } & \multirow[t]{2}{*}{ SD } & \multirow[t]{2}{*}{$\mathrm{CV}$} & \multirow[t]{2}{*}{ Range } & \multirow[t]{2}{*}{ Kurt } & \multicolumn{2}{|c|}{ Parental Mean } & \multirow[t]{2}{*}{$p^{\mathrm{d}}$} \\
\hline & & & & & & & & Female & Male $^{c}$ & \\
\hline \multirow[t]{11}{*}{ NGP } & TI & 2009 & 107.6 & 20.9 & 0.194 & $65.1-166.80 .34$ & -0.16 & 187.8 & 80.9 & \\
\hline & & 2010 & 150.7 & 26.6 & 0.177 & $92.5-259.90 .57$ & 0.81 & 172.7 & 122.7 & \\
\hline & & 2011 & 166.5 & 25.0 & 0.150 & 112.4-232.49.35 & -0.05 & 212.9 & 124.2 & \\
\hline & & 2016 & 161.5 & 30.5 & 0.189 & $54.3-308.30 .35$ & 2.35 & 187.7 & 131.3 & 0.011 \\
\hline & $\mathrm{ZM}$ & 1999 & 90.5 & 25.1 & 0.278 & $39.5-185.50 .68$ & 0.70 & 114.4 & 117.2 & \\
\hline & & 2000 & 71.2 & 16.7 & 0.235 & $26.0-130.40 .20$ & 0.15 & 88.7 & 88.0 & \\
\hline & & 2003 & 73.7 & 16.1 & 0.219 & $22.8-117.7-0.12$ & 0.26 & 59.6 & 70.8 & \\
\hline & & 2016 & 104.0 & 26.9 & 0.259 & $33.9-183.10 .17$ & -0.06 & 70.6 & 88.0 & 0.157 \\
\hline & $\mathrm{XM}$ & 1999 & 84.2 & 22.3 & 0.265 & $39.4-173.20 .76$ & 1.09 & 101.3 & 117.2 & \\
\hline & & 2000 & 71.0 & 19.8 & 0.279 & $21.3-131.20 .22$ & 0.10 & 72.2 & 88.0 & \\
\hline & & 2003 & 79.9 & 15.3 & 0.191 & $41.4-122.90 .34$ & 0.08 & 70.2 & 70.8 & 0.170 \\
\hline \multirow[t]{11}{*}{ NSP } & TI & 2009 & 121.2 & 24.2 & 0.200 & 73.3-190.0 0.38 & -0.18 & 205.8 & 95.1 & \\
\hline & & 2010 & 194.0 & 32.5 & 0.168 & $128.4-318.3 .75$ & 1.00 & 226.7 & 159.3 & \\
\hline & & 2011 & 204.2 & 30.7 & 0.150 & $141.8-298.8 .59$ & 0.01 & 248.4 & 165.0 & \\
\hline & & 2016 & 188.6 & 35.9 & 0.191 & $87.6-351.20 .65$ & 1.88 & 225.9 & 141.6 & 0.002 \\
\hline & $\mathrm{ZM}$ & 1999 & 129.7 & 31.6 & 0.244 & $61.9-242.20 .66$ & 0.75 & 126.2 & 138.5 & \\
\hline & & 2000 & 119.4 & 22.0 & 0.185 & $68.7-191.20 .33$ & -0.03 & 118.4 & 123.3 & \\
\hline & & 2003 & 105.9 & 21.6 & 0.204 & $57.1-175.40 .46$ & 0.28 & 88.3 & 88.3 & \\
\hline & & 2016 & 132.0 & 28.2 & 0.213 & $62.5-226.00 .40$ & 0.18 & 105.4 & 112.9 & 0.094 \\
\hline & $\mathrm{XM}$ & 1999 & 121.8 & 27.5 & 0.226 & $56.9-203.40 .46$ & 0.20 & 117.4 & 138.5 & \\
\hline & & 2000 & 113.6 & 20.9 & 0.184 & $56.0-193.60 .32$ & 0.97 & 101.2 & 123.3 & \\
\hline & & 2003 & 105.8 & 20.2 & 0.191 & $60.4-164.00 .40$ & -0.17 & 99.2 & 88.3 & 0.423 \\
\hline \multirow[t]{11}{*}{$\mathrm{SF}$} & TI & 2009 & 89.0 & 4.9 & 0.055 & $70.1-96.2-1.05$ & 1.28 & 91.4 & 88.7 & \\
\hline & & 2010 & 77.9 & 7.3 & 0.093 & $58.8-91.4-0.55$ & -0.10 & 75.8 & 79.1 & \\
\hline & & 2011 & 81.8 & 7.0 & 0.085 & $60.5-94.7-0.46$ & -0.23 & 85.7 & 79.8 & \\
\hline & & 2016 & 85.9 & 6.5 & 0.076 & $63.4-96.3-0.95$ & 0.82 & 82.9 & 86.4 & 0.856 \\
\hline & $\mathrm{ZM}$ & 1999 & 69.9 & 10.1 & 0.145 & $40.7-92.6-0.12$ & -0.26 & 90.7 & 84.6 & \\
\hline & & 2000 & 59.9 & 10.5 & 0.175 & $29.6-89.1-0.33$ & 0.22 & 73.4 & 70.8 & \\
\hline & & 2003 & 69.8 & 9.4 & 0.134 & $38.4-92.0-0.43$ & 0.17 & 67.5 & 80.1 & \\
\hline & & 2016 & 78.4 & 9.1 & 0.116 & $42.0-94.5-0.99$ & 1.01 & 75.7 & 79.1 & 0.682 \\
\hline & $\mathrm{XM}$ & 1999 & 69.7 & 11.7 & 0.168 & $34.8-90.3-0.37$ & -0.52 & 86.3 & 84.6 & \\
\hline & & 2000 & 62.1 & 11.9 & 0.191 & $28.7-89.4-0.36$ & 0.00 & 71.6 & 70.8 & \\
\hline & & 2003 & 75.9 & 7.6 & 0.100 & $53.7-93.9-0.34$ & -0.30 & 70.6 & 80.1 & 0.579 \\
\hline
\end{tabular}

a NGP, number of grains per panicle; NSP, number of spikelets per panicle; SF, spikelet fertility. ${ }^{\mathrm{b}} \mathrm{TI}$, Teqing/IRBB near isogenic lines, including 122 of Teqing/IRBB52, 77 of Teqing/IRBB59, two of Teqing/IRBB50, and each of Teqing/IRBB51, Teqing/IRBB54 and Teqing/IRBB55; ZM, Zhenshan 97/Milyang 46; XM, Xieqingzao/Milyang46. ${ }^{c}$ Measured as the mean value of IRBB52 and IRBB59 in the TI population. ${ }^{d}$ Two-tailed $p$ value of Student's $t$ test. 
NGP was positively correlated with NSP and SF in all three RIL populations but the coefficients were obviously higher between NGP and NSP than between NGP and SF in each population (Figure 2). Non-significant correlation between NSP and SF was detected in three RIL populations except in TI.
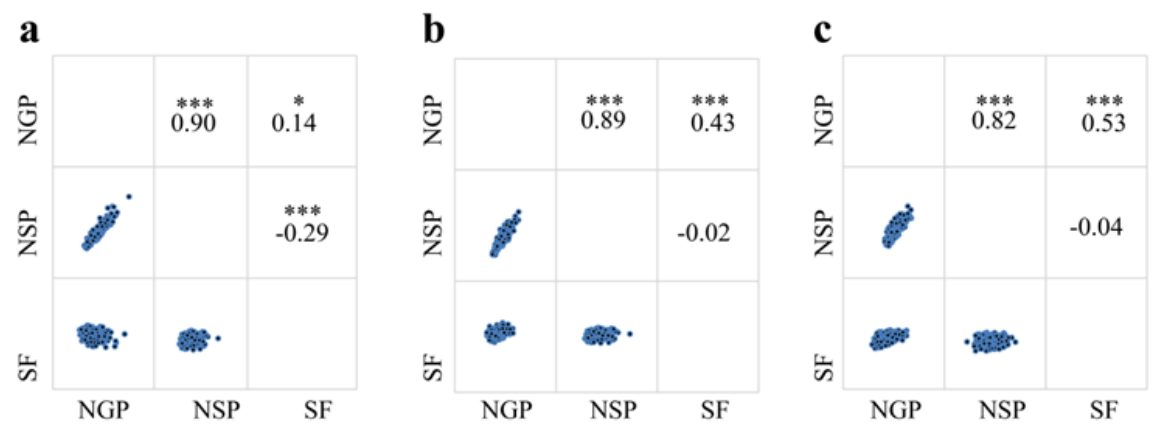

Figure 2. Correlation between different traits using mean value in Teqing/IRBB lines (TI) population (a), Zhenshan 97/Milyang 46 (ZM) population (b) and Xieqingzao/Milyang 46 (XM) population (c). The upper panel contains the correlation coefficients, and the lower panel contains the distributions of the three traits. ${ }^{*}$ and ${ }^{* * *}$ represent significant level at 0.05 and 0.001 , respectively.

In TI, the standard deviations of NGP were 20.9, 26.6, 25.0, and 30.5 in 2009, 2010, 2011, and 2016, respectively (Table 1), while the standard deviation values of the Ti52-2 originated from one RH of TI were reduced to 17.0 and 12.3 in 2016 and 2017, respectively (Table S2). Furthermore, the standard deviation values of ZC5, ZC9 and ZC12 derived from three RHs of Ti52-2 were decreased to 8.5, 9.6 and 9.9 in 2017, respectively (Table S2). Likewise, the coefficients of variation decreased from 0.150 0.194 to 0.118 and 0.079 , then to $0.056 \sim 0.063$ (Table 1 and Table S2). A similar trend was also observed between TI and Ti52-2 for NSP. These results were in line with the expectation that the background variations get more homozygous from TI to Ti52-2 and then to ZC5, ZC9, and ZC12.

\subsection{QTLs Detected in Three RIL Populations}

A total of 58 QTLs were detected in three RIL populations and distributed on all 12 rice chromosomes, including 22 for NGP, 17 for NSP, and 19 for SF (Table 2). 
Table 2. Quantitative trait locus (QTLs) detected for NGP, NSP, and SF in three RIL populations.

\begin{tabular}{|c|c|c|c|c|c|c|c|c|c|c|c|c|c|}
\hline Trait & $\mathrm{QTL}^{\mathrm{a}}$ & TI Population & & & & ZM Population & & & & XM Population & & & \\
\hline & & Interval & $L O D$ & $A^{\mathrm{b}}$ & $R^{2}(\%)^{\mathrm{C}}$ & Interval & $L O D$ & $A$ & $R^{2}(\%)$ & Interval & $L O D$ & $A$ & $R^{2}(\%)$ \\
\hline \multirow[t]{18}{*}{ NGP } & qNGP1.1 & & & & & RG532-RM151 & 29.1 & 6.13 & 15.2 & RG532-RM1195 & 10.1 & 4.32 & 6.6 \\
\hline & $q N G P 1.2$ & Wn34352-RM11869 & 4.9 & -3.17 & 1.9 & RM315-RZ538 & 7.4 & 2.66 & 2.5 & & & & \\
\hline & $q N G P 2.2$ & RM6-RM240 & 48.9 & -10.78 & 20.6 & & & & & & & & \\
\hline & qNGP3.1 & RM15303-RM16 & 25.9 & -7.90 & 11.4 & & & & & & & & \\
\hline & $q N G P 3.2$ & & & & & & & & & RM85-RG418A & 5.7 & -3.60 & 4.6 \\
\hline & qNGP4 & RM349-RM3333 & 8.7 & 4.00 & 3.4 & RG776A-RG620 & 5.4 & -2.60 & 2.9 & & & & \\
\hline & $\begin{array}{l}\text { qNGPb.1 } \\
\text { qNGP5.2 }\end{array}$ & RM146-RM164 & 7.5 & 3.25 & 2.9 & RG13-RM164 & 5.5 & -2.72 & 2.6 & CDO82-RG182 & 4.6 & -2.78 & 2.6 \\
\hline & $q N G P 5.3$ & & & & & RG573-RG470 & 5.8 & -2.70 & & & & & \\
\hline & qNGP5.4 & RM274-RM334 & 9.3 & 3.81 & 3.2 & & & & & & & & \\
\hline & $q N G P 6.1$ & & & & & RM508-RM190 & 6.8 & 2.19 & 4.4 & & & & \\
\hline & $q N G P 6.2$ & & & & & RZ398-RM204 & 19.2 & -4.46 & 12.4 & RZ398-RM217 & 6.5 & -3.16 & 3.6 \\
\hline & $q N G P 6.3$ & RM276-RM549 & 9.2 & -4.43 & 3.9 & & & & & & & & \\
\hline & $q N G P 6.4$ & RM3827-RM20361 & 4.7 & -2.69 & 1.5 & & & & & & & & \\
\hline & $q N G P 7.1$ & & & & & RM3859-RG678 & 9.3 & 2.54 & 5.4 & & & & \\
\hline & $q N G P 7.2$ & RM70-RM18 & 7.8 & -3.97 & 2.7 & & & & & & & & \\
\hline & qNGP9.1 & RM8206-RM219 & 5.8 & -3.00 & 1.6 & & & & & & & & \\
\hline & $q N G P 9.2$ & & & & & RM242-RM108 & 5.4 & 2.58 & 2.5 & & & & \\
\hline & $q N G P 11$ & & & & & RG167-RM287 & 6.5 & 2.99 & 2.9 & & & & \\
\hline \multirow[t]{17}{*}{ NSP } & $q N S P 1$ & & & & & RG532-RM151 & 33.6 & 7.41 & 15.0 & RG532-RM1195 & 15.4 & 6.60 & 11.1 \\
\hline & qNSP2.1 & RM3732-RM71 & 4.8 & 3.67 & 1.8 & & & & & RZ742-RZ512 & 7.4 & -4.58 & 5.2 \\
\hline & $q N S P 2.2$ & RM6-RM240 & 53.7 & -13.37 & 22.1 & RM240-RZ123 & 4.8 & -3.07 & 2.1 & & & & \\
\hline & $q N S P 2.3$ & Tw35293-RM207 & 5.2 & -3.63 & 1.9 & & & & & & & & \\
\hline & $q N S P 3$ & RM15303-RM16 & 9.0 & -5.19 & 3.3 & & & & & & & & \\
\hline & $q N S P 4$ & RM3474-RM6992 & 8.7 & 4.36 & 3.0 & RG776A-RG620 & 7.4 & -3.41 & 2.9 & & & & \\
\hline & qNSP5.1 & RM164-RM18927 & 5.4 & 3.13 & 1.9 & RM164-RM163 & 6.7 & -3.28 & 2.9 & RM163-RG470 & 4.5 & -3.44 & 2.9 \\
\hline & qNSP5.2 & & & & & RG573-RG470 & 5.1 & -2.93 & 2.7 & & & & \\
\hline & qNSP5.3 & RM274-RM334 & 7.5 & 4.27 & 2.5 & & & & & & & & \\
\hline & $q N S P 6.1$ & & & & & & & & & RZ398-RM217 & 7.5 & -3.79 & 6.5 \\
\hline & $q N S P 6.2$ & RM276-RM549 & 22.6 & -8.35 & 8.6 & RM253-RM276 & 24.3 & -7.30 & 13.6 & & & & \\
\hline & $q N S P 7.1$ & & & & & RM1243-RM3859 & 7.2 & 2.93 & 4.6 & RM1243-RM3859 & 7.9 & 3.72 & 6.4 \\
\hline & $q N S P 7.2$ & RM70-RM18 & 11.2 & -5.86 & 4.1 & & & & & RZ264-RZ626 & 5.4 & -4.03 & 3.4 \\
\hline & $q N S P 10$ & & & & & & & & & RM1859-RM184 & 5.0 & -3.42 & 4.2 \\
\hline & qNSP11.1 & & & & & RG118-RM202 & 5.1 & 2.85 & 2.0 & & & & \\
\hline & qNSP11.2 & & & & & & & & & RZ797-RG103 & 6.9 & 4.09 & 5.5 \\
\hline & $q N S P 12$ & RM511-RM28313 & 18.4 & 7.24 & 6.8 & & & & & & & & \\
\hline
\end{tabular}


Table 2. Cont

\begin{tabular}{|c|c|c|c|c|c|c|c|c|c|c|c|c|c|}
\hline Trait & QTL $^{a}$ & TI Population & & & & ZM Population & & & & XM Population & & & \\
\hline & & Interval & $L O D$ & $A^{\mathrm{b}}$ & $R^{2}(\%)^{\mathrm{c}}$ & Interval & $L O D$ & $A$ & $R^{2}(\%)$ & Interval & $L O D$ & $A$ & $R^{2}(\%)$ \\
\hline \multirow[t]{18}{*}{ SF } & $q S F 1.1$ & & & & & RM294A-RM294B & 6.6 & 1.31 & 4.8 & & & & \\
\hline & $q S F 1.2$ & RM12178-RM12210 & 7.0 & -0.95 & 3.1 & & & & & & & & \\
\hline & $q S F 2.1$ & & & & & RZ318-RM263 & 6.4 & -0.74 & 3.6 & & & & \\
\hline & $q S F 2.2$ & RM6-RM240 & 6.5 & 0.87 & 3.3 & & & & & & & & \\
\hline & qSF3.1 & RM15303-RM16 & 13.1 & -1.45 & 8.2 & & & & & & & & \\
\hline & qSF3.2 & & & & & & & & & R1927-RM143 & 4.9 & -1.25 & 3.2 \\
\hline & qSF3.3 & & & & & RZ613-RM85 & 11.4 & -1.80 & 7.4 & RM85-RG418A & 6.1 & -1.99 & 7.6 \\
\hline & $q S F 4.1$ & & & & & RM551-RM261 & 4.8 & 0.93 & 3.3 & & & & \\
\hline & $q S F 4.2$ & Fo13346-RM303 & 8.6 & -1.07 & 5.1 & & & & & & & & \\
\hline & $q S F 5.2$ & RM18038-RM18189 & 22.2 & 1.96 & 13.9 & & & & & & & & \\
\hline & $q S F 6.1$ & & & & & & & & & RM190-RZ516 & 7.3 & 1.83 & 7.9 \\
\hline & $q S F 6.2$ & RM6119-RM276 & 7.5 & 0.93 & 3.1 & RG138-RM111 & 5.3 & 0.88 & 4.2 & & & & \\
\hline & qSF6.3 & RM340-RM20731 & 9.0 & 1.19 & 5.4 & & & & & & & & \\
\hline & qSF8 & RM23001-RM210 & 6.4 & 0.95 & 3.2 & & & & & & & & \\
\hline & qSF9 & & & & & RM105-RM3700 & 7.0 & 1.35 & 4.0 & & & & \\
\hline & $q S F 10.1$ & & & & & & & & & RM3229B-RM1376 & 5.0 & 1.13 & 5.6 \\
\hline & $q S F 10.1$ & RM3773-RM3123 & 5.4 & 0.83 & 2.9 & & & & & & & & \\
\hline & $q S F 12$ & RM28313-RM28597 & 6.8 & -0.94 & 2.9 & & & & & & & & \\
\hline
\end{tabular}

${ }^{\mathrm{a}}$ QTL are named as proposed by McCouch and CGSNL (2008). ${ }^{\mathrm{b}} \mathrm{A}$, additive effect of replacing a maternal allele with a paternal allele. ${ }^{\mathrm{c}} R^{2}$, proportion of phenotypic variance explained by the QTL effect. 
For NGP, 12, 11, and four QTLs were identified in TI, ZM, and XM, respectively. Among them, five QTLs were shared between two populations and 17 were identified in single population (Table 2 and Figure 3a). In TI, qNGP2.2 and qNGP3.1 explained the highest two proportions of phenotypic variance with the value of $20.6 \%$ and $11.4 \%$, respectively. The other 10 QTLs had the phenotypic variance explanation ranging from 1.5 to $3.9 \%$, of which three QTLs: qNGP1.2, qNGP4, and qNGP5.2 were shared with ZM. In ZM, $q N G P 1.1$ and $q N G P 6.2$ were revealed to have the highest two $R^{2}$ of $15.2 \%$ and $12.4 \%$, which were also detected in XM. The $R^{2}$ values of other QTLs were much smaller ranging as 2.5-5.4\%. In XM, all four QTLs showed small $R^{2}$ values ranging from 2.6 to $6.6 \%$. No QTL was found in both of TI and XM. The overall $R^{2}$ for NGP were much higher in TI and ZM than in XM, which were $59.0 \%, 56.3 \%$, and $17.4 \%$, respectively. Significant genotype-by-environment (GE) interaction was observed for $q N G P 1.1$ and $q N G P 6.2$ only in ZM (Table S3).

a

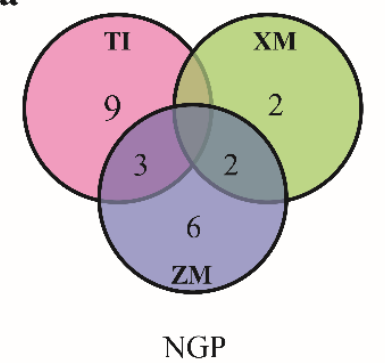

b

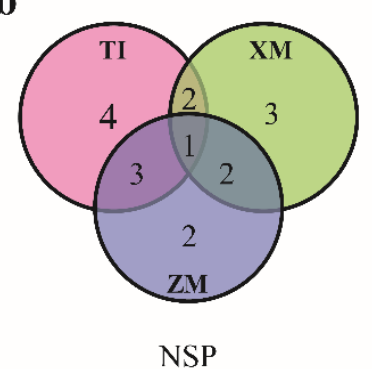

c

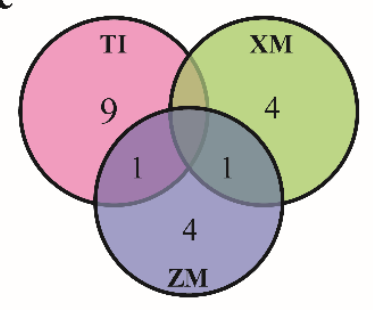

$\mathrm{SF}$

Figure 3. Number of QTLs detected in three RIL populations for NGP (a), NSP (b) and SF (c), respectively.

For NSP, ten, eight and eight QTLs were detected in TI, ZM and XM, respectively. Among them, one QTL was shared within all three populations, seven QTLs were contributed by two populations and nine QTLs were identified in single population (Table 2 and Figure 3b). qNSP5.1, the only one stably detected in three populations, lay in the interval of RM164-RM18927 on chromosome 5 in TI and showed minor effect in all populations. qNSP2.2 displayed the largest $\mathrm{R}^{2}$ of $22.1 \%$ in TI but nearly the smallest $R^{2}$ of $2.1 \%$ in $\mathrm{ZM}$, indicating divergent effects produced in different genetic background. In ZM, qNSP1 and $q N S P 6.2$ had the highest two $\mathrm{R}^{2}$ of $15.1 \%$ and $13.6 \%$, the other six QTLs had $R^{2}$ ranging from 2.0 to $4.6 \%$. In XM, qNSP1 also had the highest contribution of $11.1 \%$, and the other seven QTLs had $R^{2}$ ranging from 2.9 to $6.5 \%$. The overall $R^{2}$ of the QTLs were $55.9 \%, 45.8 \%$ and $45.1 \%$ in TI, ZM and XM, respectively. Except $q N S P 2.3, q N S P 10$, and $q N S P 11.2$, all QTLs for NSP were simultaneously shared the same intervals as QTLs for NGP, in accordance with high positive correlation between NGP and NSP in three populations. In addition, one significant GE interaction was found for $q N S P 1$ in ZM (Table S3).

For SF, ten, six and five QTLs were identified in TI, ZM and XM, respectively. Only two QTLs were shared between two populations (Table 2 and Figure 3c). qSF3.3 was simultaneously identified in $\mathrm{ZM}$ and XM, of which the $R^{2}$ reached almost the highest with value of $7.4 \%$ and $7.6 \%$, respectively. qSF6.2 was detected in TI and ZM, with minor effect to SF in both populations. In TI, $q S F 5.2$ appeared the highest $R^{2}$ of $13.9 \%$ and the remaining 16 QTLs had the $R^{2}$ ranging from 2.9 to $8.2 \%$. The overall $R^{2}$ of the QTLs were $51.1 \%, 27.3 \%$ and $30.1 \%$ in TI, ZM and XM, respectively. Only one QTL, $q S F 2.1$, showed significant GE interaction in ZM (Table S3).

\subsection{QTLs Detected in Ti52-2 Population}

A total of five, three and four QTLs for NGP, NSP, and SF were detected in Ti52-2 population, respectively and distributed on rice chromosome 3, 5, 6, 9, 11 and 12 (Table 3). No significant GE interaction was observed. 
Table 3. QTLs detected for NGP, NSP, and SF in Ti52-2 population.

\begin{tabular}{|c|c|c|c|c|c|}
\hline Trait & QTL & Interval & $L O D$ & $A^{\mathrm{a}}$ & $R^{2}(\%){ }^{b}$ \\
\hline \multirow[t]{5}{*}{ NGP } & qNGP3.3 & RM232 & 3.2 & 2.29 & 4.1 \\
\hline & qNGP5.5 & RM18927-RM3321 & 4.1 & -2.46 & 4.9 \\
\hline & qNGP6.1 & RM469-RM589 & 5.8 & 2.31 & 9.9 \\
\hline & qNGP9.1 & RM5688-RM219 & 3.5 & -2.18 & 5.6 \\
\hline & qNGP12.1 & Pita-RM511 & 3.1 & 2.19 & 4.8 \\
\hline \multirow[t]{3}{*}{ NSP } & qNSP3.2 & RM232 & 2.6 & 1.89 & 2.2 \\
\hline & qNSP5.4 & RM18927-RM3321 & 5.5 & -3.44 & 5.3 \\
\hline & qNSP12 & RM3246-Pita & 6.8 & 3.73 & 7.8 \\
\hline \multirow[t]{4}{*}{ SF } & $q S F 3.4$ & RM14303-RM14383 & 3.8 & 0.53 & 7.3 \\
\hline & qSF6.1 & RM587-RM584 & 7.8 & 1.08 & 16.3 \\
\hline & qSF9.2 & RM5688-RM219 & 6.1 & -0.98 & 11.3 \\
\hline & $q S F 11$ & RM224-RM5926 & 6.0 & 0.97 & 9.0 \\
\hline
\end{tabular}

a $\mathrm{A}$, additive effect of replacing a maternal allele with a paternal allele. ${ }^{\mathrm{b}} R^{2}$, proportion of phenotypic variance explained by the QTL effect.

Ti52-2 was derived from TI carrying 13 segregating regions (Figure 4). In Ti52-2, those regions involving QTLs with major effects for NGP, NSP, and SF in TI and other two RIL populations had become homozygous, such as RG532-RM151 interval containing qNGP1.1 and qNSP1, RM6-RM240 interval containing $q N G P 2.2$ and $q N S P 2.2$, RM15303-RM16 interval containing $q N G P 3.1$ and $q S F 3.1$, RM276-RM549 interval containing qNGP6.2 and qNSP6.2, RM18038-RM18189 interval containing qSF5.2. Therefore, Ti52-2 is a good candidate to detect minor-effect QTL for NGP, NSP, and SF.
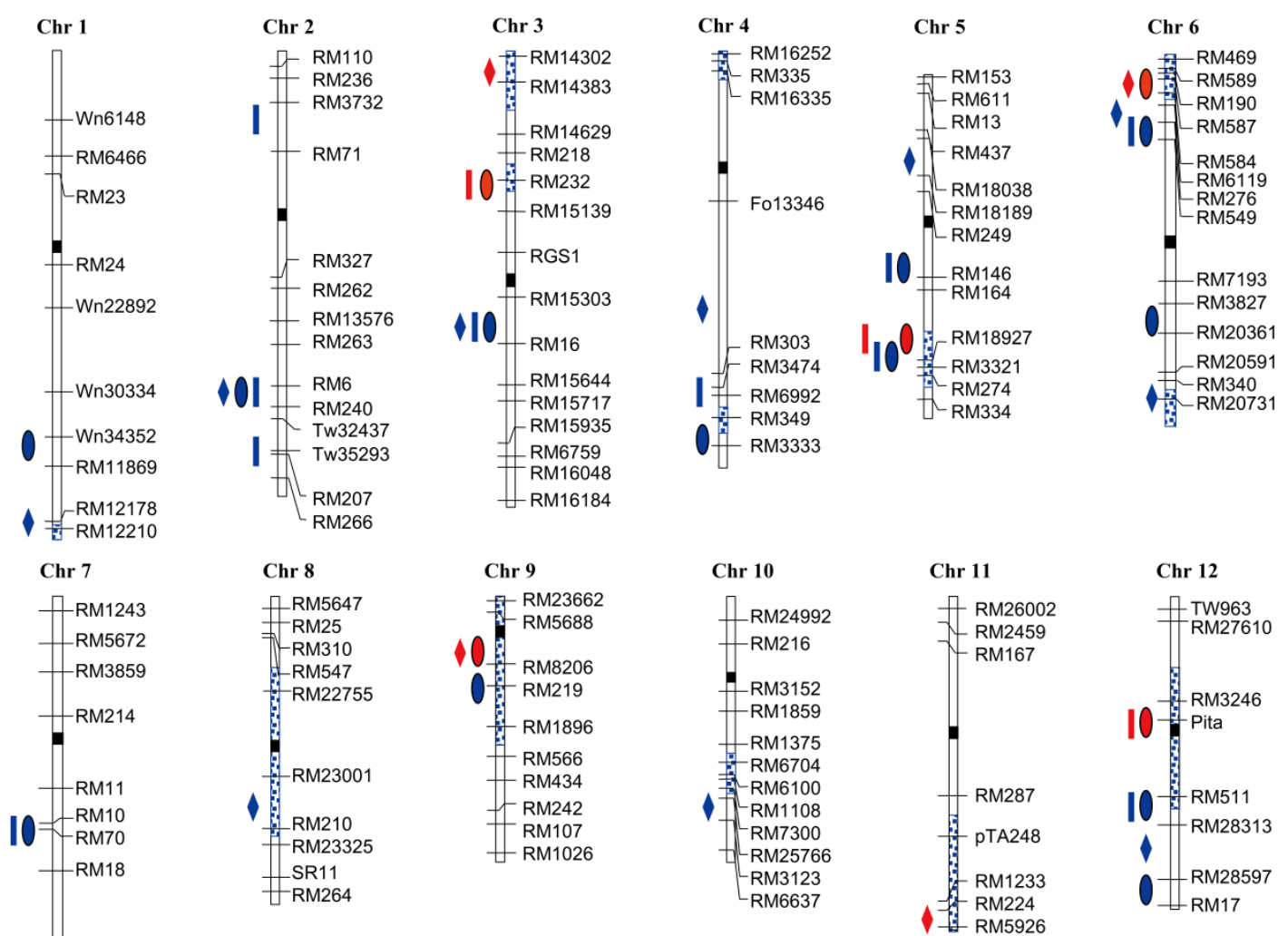

П Homozygous region in Ti52-2

QTL for NGP detected in Ti52-2

QTL for NSP detected in Ti52-2 $\$ QTL for SF detected in Ti52-2

Segregating region in Ti52-2

QTL for NGP detected in TI

QTL for NSP detected in TI

$\checkmark$ QTL for SF detected in TI

Figure 4. Distribution of QTLs for NGP, NSP, and SF detected in Ti52-2 and TI populations. 
Both of $q$ NGP9.1 and $q$ NGP12.1 were validated in Ti52-2 and the direction of additive effects remained unchanged between TI and Ti52-2. Moreover, the $R^{2}$ increased from 1.6 to $5.6 \%$ and 3.9 to $4.8 \%$, respectively. In TI, qNGP5.4 was detected in the interval of RM274-RM334 on chromosome 5 and the IRBB52 allele increased NGP 3.81. In Ti52-2, the locus of RM334 had got homozygous, while RM274 remained be segregating. However, a QTL was found in the interval of RM18927-RM3321 upstream of $q N G P 5.4$ with the TQ allele increasing NGP 2.46. Hence, the new QTL was named qNGP5.5. Among the five QTLs for NGP detected in Ti52-2, qNGP6.1 with the highest $R^{2}$ was undetected previously in TI but mapped in the similar interval of RM508-RM190 in ZM, while $q N G P 3.3$ with the lowest $R^{2}$ was not identified in previous three RIL populations.

Amid of the three QTLs for NSP detected in Ti52-2, qNSP12 was validated with the largest $R^{2}$ of 7.8\% and the enhancing allele was from IRBB52 in both TI and Ti52-2. qNSP5.4, a new QTL with an allelic effect in opposite direction to qNSP5.3 in the interval of RM274-RM334 detected in TI, was detected in the RM18927-RM3321 region. In addition, qNSP3.2 having the marginal LOD threshold and the smallest $R^{2}$ was not previously detected in TI, ZM and XM.

Out of the four QTLs for SF detected in Ti52-2, qSF6.1 with the highest $R^{2}$ of $16.3 \%$ was mapped in RM586-RM584 region, which was undetected in TI and lay in a similar interval of RM190-RZ516 detected in XM. The others, qSF9.2, qSF3.4, and qSF11, were not identified previously in three RIL populations. Either position or additive effect direction of $q S F 9.2$ was different from $q S F 9$ identified in ZM. Thus $q S F 9.2$ was a new QTL and $q S F 9$ was renamed as $q S F 9.1$.

\subsection{Validation of QTLs for NGP in ZC5, ZC9, and ZC12 Populations}

The three QTLs, qNGP5.5, qNGP9.1 and NGP12.1, which had shown significant effects in Ti52-2, were well validated in the new secondary segregation population, respectively (Table 4).

Table 4. QTLs detected for NGP in the ZC5, ZC9, and ZC12 populations.

\begin{tabular}{ccccccc}
\hline QTL & Population & Interval & LOD & $A^{\mathbf{a}}$ & $\boldsymbol{D}^{\boldsymbol{b}}$ & $\boldsymbol{R}^{\mathbf{2}} \mathbf{( \% )}$ \\
\hline qNGP5.5 & ZC5 S $_{1: 2}$ & RM18927-RM3321 & 2.72 & -1.94 & 3.42 & 6.3 \\
qNGP9.1 & ZC9 S $1: 2_{1: 3}$ & RM219-RM1896 & 2.22 & -2.96 & 1.00 & 5.1 \\
qNGP12.1 & ZC12 S $1: 2^{\mathbf{c}}$ & RM3246-Pita & 9.06 & 5.68 & 0.43 & 16.0 \\
\hline
\end{tabular}

a $\mathrm{A}$, additive effect of replacing a maternal allele with a paternal allele. ${ }^{\mathrm{b}} D$, dominance effect. ${ }^{\mathrm{c}} R^{2}$, proportion of phenotypic variance explained by the QTL effect.

For $q N G P 5.5$ and $q N G P 9.1$, TQ allele increased NGP about 2.46 and 2.18 and explained $4.9 \%$ and $5.6 \%$ of phenotypic variance in Ti52-2, respectively. In ZC5 and ZC9, TQ allele also increased NGP about 1.94 and 2.96 , explaining $6.3 \%$ and $5.1 \%$ of phenotypic variance, respectively. For $q N G P 12.1$, the additive effect was doubled and the $R^{2}$ values were increased by 3.3 times in ZC12.

\section{Discussion}

Grain number is one of the most important agronomic traits in the determination of rice productivity, which is governed by multiple QTLs with major or minor effect. In the present study, a total of 58 QTLs were detected in three RIL populations, including 22, 17, and 19 for NGP, NSP, and SF, respectively. The individual QTL counted for $1.5 \%$ to $22.1 \%$ of phenotypic variation. Among 58 QTLs, 15 with various effects shared by two or three populations and eight showed large effects with $R^{2}$ larger than $10 \%$. When major-effect QTLs were fixed, three minor-effect QTLs for NGP were further detected and validated using secondary segregation populations derived from one $\mathrm{RH}$ of the cross TQ/IRBB52 under increment homogeneous backgrounds.

Among the three minor-effect QTLs for NGP, qNGP9.1 and qNGP12.1 were stably identified in TI, Ti52-2, ZC9, and ZC12 populations, having the same direction of additive effects and similar value. Moreover, the regions of RM8206-RM219 containing $q N G P 9.1$ and RM511-RM28313 containing $q N G P 12.1$ were previously reported to affect NGP. $q F G 9$ and Gpp12.2 were detected in the region 
of RM219-RM342 on chromosome 9 and RG869-RM277 on chromosome 12, respectively [49,50]. It proves that $q N G P 9.1$ and $q N G P 12.1$ are good targets for underlying the genetic basis of grain number.

qNGP5.5 was a new QTL identified in Ti52-2. This QTL was well verified in ZC5, the direction of allelic effect remaining consistent and $R^{2}$ increased from 4.9 to $6.3 \%$. Actually, qNGP5.4 was detected in TI with an allelic effect in the opposite direction to qNGP5.5. Comparing the two loci, we found that $q$ NGP5.5 was located in RM18927-RM3321, while the qNGP5.4 was detected in RM274-RM334. The segregation region of Ti52-2 population covered RM274 but uncovered RM334. In previous studies, two QTLs with opposite allelic direction for grain weight were detected in RM18927-RM334 [37,51]. Hence, there might be two tightly linked QTLs with opposite direction of additive effect in the region of RM18927-RM334.

Verification of three minor-effect QTLs was benefited from the fixation of the major-effect QTLs and increment homozygous of genetic background. Along with the background became more homogenous from TI to Ti52-2 then to ZC5, ZC9 and ZC12, the phenotypic variations were getting smaller, and the power of detection of QTLs was further improved. For instance, the standard deviation values and coefficient of variation of NGP and NSP has been decreasing with background became more homogenous (Table 1 and Table S2). Out of the 12 QTLs for NGP detected in TI, only qNGP4, qNGP5.4, $q N G P 9.1$ and $q N G P 12.1$ were located in the segregation region of Ti52-2. Except $q N G P 4$ with the smallest $R^{2}$, other three QTLs were detected in Ti52-2. In addition, two more QTLs qNGP3.3 and $q N G P 5.5$ were detected in Ti52-2. These results supported that RHs are efficient materials in the establishment of NILs and secondary mapping populations for QTL validation and fine-mapping especially for minor-effect QTLs.

Interestingly, among the five QTLs for NGP identified in Ti52-2, qNGP3.3, qNGP5.5, and qNGP12.1 were located in the same regions with $q N S P 3.2, q N S P 5.4$, and $q N S P 12$, respectively. The regions of qNGP6.1 and qNGP9.1 showed the same intervals with qSF6.1 and qSF9.2. The results suggest that the five QTLs for NGP detected in Ti52-2 is a result of the integration of NSP and SF. Usually, NGP is determined by NSP multiply SF. Thus, these QTL regions sharing for both NGP and NSP or SF might be due to a pleiotropic effect of one QTL rather than the close linkage of different QTLs.

The eight QTLs with large effects detected in three RIL populations were located in six regions on chromosome 1, 2, 3, 5 and 6. Except for qSF5.2, all QTLs controlled NGP and/or NSP.

The region of RG532-RM151 on chromosome 1 containing $q N G P 1.1$ and $q N S P 1$ showed the largest $\mathrm{R}^{2}$ for NGP and NSP in both of ZM and XM, while the interval of RM6-RM240 on chromosome 2 covering $q N G P 2.2$ and $q N S P 2.2$ showed the largest $R^{2}$ for NGP and NSP in TI. The special significance demonstrated in the given population might reveal the different mechanism of variant ecological type of rice varieties. Gn1a, the first cloned QTL for grain number in rice, is located in the former region [18], whereas no QTL for grain number was found to be fine-mapped or cloned in the latter interval. By comparing the physical position, we found a heading date QTL, DTH2, situated within this interval [52]. Anyway, no QTL controlling heading date was detected around this region in previous studies [42], which indicated that DTH2 could not the candidate gene of $q N G P 2.2$ and $q N S P 2.2$. In addition, we found that GS2, a major QTL for grain size was lain $0.7 \mathrm{Mb}$ upstream of RM6. Hu et al. reported that no significant differences of NGP were found between the recurrent parents and NILs [53], suggesting it would not probably be the pleiotropic effect of GS2. Hence, this locus might be a new QTL controlling the grain number. Fine-mapping of $q N G P 2.2$ is to be undertaken.

The region of RM15303-RM16 on chromosome 3 was another one for both of NGP and NSP identified in TI. However, it presented the second largest $\mathrm{R}^{2}$ for NGP, but much smaller effect on NSP. GL3.1, a cloned QTL for grain length, was positioned $1.9 \mathrm{cM}$ downstream of RM16 and was reported to have a significant effect on NGP [54]. More work is needed to confirm whether this locus is a new QTL for grain number or just a pleiotropic effect of GL3.1.

The region of RZ398-RM217 on chromosome 6 exhibited the second largest $\mathrm{R}^{2}$ for NGP but no significant effect on NSP in ZM. It also displayed minor effect on NGP and NSP in XM. No QTL controlling grain number had been cloned or fine-mapped in this interval. In the other hand, the 
region of RM253-RM276 downstream of RZ398-RM217 exhibited the second largest $\mathrm{R}^{2}$ for NSP but no significant effect on NGP in ZM. It was noteworthy that it also displayed the second largest $\mathrm{R}^{2}$ for NSP and minor effect on NGP in TI. It was previously reported that a QTL controlled NGP and yield-related traits in the same region [55]. Hence, these QTLs provided new candidates for gene cloning and marker-assisted breeding.

\section{Conclusions}

A total of 58 QTLs for NGP, NSP, and SF were detected in three RIL populations of indica rice. Among them, eight QTLs showed large effects with $R^{2}$ larger than $10 \%$. Three QTLs with minor effects for NGP, qNGP5.5, qNGP9.1, and qNGP12.1, were further detected and validated using segregation populations derived from one RH of the cross TQ/IRBB52. These results proved that the use of residual heterozygotes to construct secondary mapping populations is an efficient strategy to detect minor-effect QTLs for complex traits. The results also enrich our understanding of the mechanism of grain number and provide foundation for cloning and selecting candidate for marker-assisted selection breeding in rice.

Supplementary Materials: The following are available online at http://www.mdpi.com/2073-4395/10/2/180/s1, Table S1: DNA markers used in the ZC5 population. Table S2: Phenotypic performance of NGP and NSP in the Ti52-2, ZC5, ZC9 and ZC12 populations. Table S3: Genotype-by-environment interaction (GE) detected in the RIL populations.

Author Contributions: J.Z. and Y.F. conceived and designed the experiments; X.N., Y.Z. and Z.S. performed the experiments; S.Y. and J.Z. analyzed the data; X.N. and Y.F. wrote the paper.

Funding: This research was supported by grants from the National Key Research and Development Program of China (2017YFD0100305), and the National Natural Science Foundation of China (31521064).

Conflicts of Interest: The authors declare no conflict of interest.

\section{References}

1. Zhang, H.; Chen, T.T.; Liu, L.J.; Wang, Z.Q.; Yang, J.C.; Zhang, J.H. Performance in grain yield and physiological traits of rice in the Yangtze River Basin of China during the last 60 yr. J. Integr. Agric. 2013, 12, 57-66. [CrossRef]

2. Yang, S.H.; Cheng, B.Y.; Shen, W.F.; Liao, X.Y. Progress and strategy of the improvement of indica rice varieties in the Yangtse valley of China. Chin. J. Rice Sci. 2004, 18, 89-93.

3. Xing, Y.; Zhang, Q. Genetic and molecular bases of rice yield. Annu. Rev. Plant Biol. 2010, 61, 421-442. [CrossRef] [PubMed]

4. Bai, X.; Wu, B.; Xing, Y. Yield-related QTLs and their applications in rice genetic improvement. J. Integr. Plant Biol. 2012, 54, 300-311. [CrossRef] [PubMed]

5. Li, J.X.; Yu, S.B.; Xu, C.G.; Tan, Y.F.; Gao, Y.J.; Li, X.H.; Zhang, Q. Analyzing quantitative trait loci for yield using a vegetatively replicated $\mathrm{F}_{2}$ population from a cross between the parents of an elite rice hybrid. Theor. Appl. Genet. 2000, 101, 248-254. [CrossRef]

6. Yu, S.B.; Li, J.X.; Xu, C.G.; Tan, Y.F.; Gao, Y.J.; Li, X.H.; Zhang, Q.; Saghai Maroof, M.A. Importance of epistasis as the genetic basis of heterosis in an elite rice hybrid. Proc. Natl. Acad. Sci. USA 1997, 94, 9226-9231. [CrossRef]

7. Hittalmani, S.; Shashidhar, H.E.; Bagali, P.G.; Huang, N.; Sidhu, J.S.; Singh, V.P.; Khush, G.S. Molecular mapping of quantitative trait loci for plant growth, yield and yield related traits across three diverse locations in a doubled haploid rice population. Euphytica 2002, 125, 207-214. [CrossRef]

8. Lu, C.; Shen, L.; Tan, Z.; Xu, Y.; He, P.; Chen, Y.; Zhu, L. Comparative mapping of QTLs for agronomic traits of rice across environments by using a doubled-haploid population. Theor. Appl. Genet. 1997, 94, 145-150. [CrossRef]

9. Xiao, J.; Li, J.; Yuan, L.; Tanksley, S.D. Identification of QTLs affecting traits of agronomic importance in a recombinant inbred population derived from a subspecific rice cross. Theor. Appl. Genet. 1996, 92, 230-244. [CrossRef] 
10. Zhuang, J.Y.; Fan, Y.Y.; Rao, Z.M.; Wu, J.L.; Xia, Y.W.; Zheng, K.L. Analysis on additive effects and additive-by-additive epistatic effects of QTLs for yield traits in a recombinant inbred line population of rice. Theor. Appl. Genet. 2002, 105, 1137-1145. [CrossRef]

11. Liu, T.; Mao, D.; Zhang, S.; Xu, C.; Xing, Y. Fine mapping SPP1, a QTL controlling the number of spikelets per panicle, to a BAC clone in rice (Oryza sativa). Theor. Appl. Genet. 2009, 118, 1509-1517. [CrossRef] [PubMed]

12. Zhang, Y.; Luo, L.; Liu, T.; Xu, C.; Xing, Y. Four rice QTL controlling number of spikelets per panicle expressed the characteristics of single Mendelian gene in near isogenic backgrounds. Theor. Appl. Genet. 2009, 118, 1035-1044. [CrossRef] [PubMed]

13. Tian, F.; Zhu, Z.; Zhang, B.; Tan, L.; Fu, Y.; Wang, X.; Sun, C.Q. Fine mapping of a quantitative trait locus for grain number per panicle from wild rice (Oryza rufipogon Griff.). Theor. Appl. Genet. 2006, 113, 619-629. [CrossRef] [PubMed]

14. Shan, J.X.; Zhu, M.Z.; Shi, M.; Gao, J.P.; Lin, H.X. Fine mapping and candidate gene analysis of spd6, responsible for small panicle and dwarfness in wild rice (Oryza rufipogon Griff.). Theor. Appl. Genet. 2009, 119, 827-836. [CrossRef]

15. Hu, Z.; Cao, L.; Sun, X.; Zhu, Y.; Zhang, T.; Jiang, L.; Liu, Y.; Dong, S.; Sun, D.; Yang, J.; et al. Fine mapping of a major quantitative trait locus, qgnp7 $(t)$, controlling grain number per panicle in African rice (Oryza glaberrima S.). Breed. Sci. 2018, 68, 606-613. [CrossRef] [PubMed]

16. Zhou, Y.; Tao, Y.; Yuan, Y.; Zhang, Y.; Miao, J.; Zhang, R.; Yi, C.; Gong, Z.; Yang, Z.; Liang, G. Characterisation of a novel quantitative trait locus, GN4-1, for grain number and yield in rice (Oryza sativa L.). Theor. Appl. Genet. 2018, 131, 637-648. [CrossRef] [PubMed]

17. Singh, V.K.; Ellur, R.K.; Singh, A.K.; Nagarajan, M.; Singh, B.D.; Singh, N.K. Effect of $q$ GN4.1 QTL for grain number per panicle in genetic backgrounds of twelve different mega varieties of rice. Rice 2018, 11, 8. [CrossRef]

18. Ashikari, M.; Sakakibara, H.; Lin, S.; Yamamoto, T.; Takashi, T.; Nishimura, A.; Angeles, E.R.; Qian, Q.; Kitano, K.; Matsuoka, M. Cytokinin oxidase regulates rice grain production. Science 2005, 309, 741-745. [CrossRef]

19. Huo, X.; Wu, S.; Zhu, Z.; Liu, F.; Fu, Y.; Cai, H.; Sun, X.; Gu, P.; Xie, D.; Tan, L.; et al. NOG1 increases grain production in rice. Nat. Commun. 2017, 8, 1497. [CrossRef]

20. Huang, X.; Qian, Q.; Liu, Z.; Sun, H.; He, S.; Luo, D.; Xia, G.; Chu, C.; Liu, J.; Fu, X. Natural variation at the DEP1 locus enhances grain yield in rice. Nat. Genet. 2009, 41, 494-497. [CrossRef]

21. Jiao, Y.; Wang, Y.; Xue, D.; Wang, J.; Yan, M.; Liu, G.; Dong, G.; Zeng, D.; Lu, Z.; Zhu, X.; et al. Regulation of OsSPL14 by OsmiR156 defines ideal plant architecture in rice. Nat. Genet. 2010, 42, 541-544. [CrossRef] [PubMed]

22. Miura, K.; Ikeda, M.; Matsubara, A.; Song, X.J.; Ito, M.; Asano, K.; Matsuoka, M.; Kitano, H.; Ashikari, M. OsSPL14 promotes panicle branching and higher grain productivity in rice. Nat. Genet. 2010, 42, 545-549. [CrossRef] [PubMed]

23. Terao, T.; Nagata, K.; Morino, K.; Hirose, T. A gene controlling the number of primary rachis branches also controls the vascular bundle formation and hence is responsible to increase the harvest index and grain yield in rice. Theor. Appl. Genet. 2010, 120, 875-893. [CrossRef] [PubMed]

24. Ookawa, T.; Hobo, T.; Yano, M.; Murata, K.; Ando, T.; Miura, H.; Asano, K.; Ochiai, Y.; Ikeda, M.; Nishitani, R.; et al. New approach for rice improvement using a pleiotropic QTL gene for lodging resistance and yield. Nat. Commun. 2010, 1, 132. [CrossRef]

25. Wu, Y.; Wang, Y.; Mi, X.F.; Shan, J.X.; Li, X.M.; Xu, J.L.; Lin, H.X. The QTL GNP1 encodes GA20ox1, which increases grain number and yield by increasing cytokinin activity in rice panicle meristems. PLoS Genet. 2016, 12, e1006386. [CrossRef]

26. Fujita, D.; Trijatmiko, K.R.; Tagle, A.G.; Sapasap, M.V.; Koide, Y.; Sasaki, K.; Tsakirpaloglou, N.; Gannaban, R.B.; Nishimura, T.; Yanagihara, Y.; et al. NAL1 allele from a rice landrace greatly increases yield in modern indica cultivars. Proc. Natl. Acad. Sci. USA 2013, 110, 20431-20436. [CrossRef]

27. Zhang, G.H.; Li, S.Y.; Wang, L.; Ye, W.J.; Zeng, D.L.; Rao, Y.C.; Peng, Y.L.; Hu, J.; Yang, Y.L.; Xu, J.; et al. LSCHL4 from Japonica cultivar, which is allelic to NAL1, increases yield of indica super rice 93-11. Mol. Plant 2014, 7, 1350-1364. [CrossRef] 
28. Xue, W.; Xing, Y.; Weng, X.; Zhao, Y.; Tang, W.; Wang, L.; Zhou, H.; Yu, S.; Xu, C.; Li, X.; et al. Natural variation in Ghd7 is an important regulator of heading date and yield potential in rice. Nat. Genet. 2008, 40, 761-767. [CrossRef]

29. Weng, X.; Wang, L.; Wang, J.; Hu, Y.; Du, H.; Xu, C.; Xing, Y.; Li, X.; Xiao, J.; Zhang, Q. Grain number, plant height, and heading date 7 is a central regulator of growth, development, and stress response. Plant Physiol. 2014, 164, 735-747. [CrossRef]

30. Kinoshita, N.; Kato, M.; Koyasaki, K.; Kawashima, T.; Nishimura, T.; Hirayama, Y.; Takamure, I.; Sato, T.; Kato, K. Identification of quantitative trait loci for rice grain quality and yield-related traits in two closely related Oryza sativa L. subsp. Japonica cultivars grown near the northernmost limit for rice paddy cultivation. Breed. Sci. 2017, 67, 191-206. [CrossRef]

31. Yamamoto, T.; Yonemaru, J.; Yano, M. Towards the understanding of complex traits in rice: Substantially or superficially? DNA Res. 2009, 16, 141-154. [CrossRef] [PubMed]

32. Nagata, K.; Ando, T.; Nonoue, Y.; Mizubayashi, T.; Kitazawa, N.; Shomura, A.; Matsubara, K.; Ono, N.; Mizobuchi, R.; Shibaya, T.; et al. Advanced backcross QTL analysis reveals complicated genetic control of rice grain shape in a japonica $\times$ indica cross. Breed. Sci. 2015, 65, 308-318. [CrossRef] [PubMed]

33. Chen, J.Y.; Guo, L.; Ma, H.; Chen, Y.Y.; Zhang, H.W.; Ying, J.Z.; Zhuang, J.Y. Fine mapping of $q H d 1$, a minor heading date QTL with pleiotropism for yield traits in rice (Oryza sativa L.). Theor. Appl. Genet. 2014, 127, 2515-2524. [CrossRef] [PubMed]

34. Zhu, Y.J.; Fan, Y.Y.; Wang, K.; Huang, D.R.; Liu, W.Z.; Ying, J.Z.; Zhuang, J.Y. Rice flowering locus T1 plays an important role in heading date influencing yield traits in rice. Sci. Rep. 2017, 7, 4918. [CrossRef]

35. Wang, W.; Wang, L.; Zhu, Y.; Fan, Y.; Zhuang, J. Fine-mapping of $q T G W 1.2 a$, a quantitative trait locus for 1000-grain weight in rice. Rice Sci. 2019, 26, 220-228.

36. Dong, Q.; Zhang, Z.H.; Wang, L.L.; Zhu, Y.J.; Fan, Y.Y.; Mou, T.M.; Ma, L.Y.; Zhuang, J.Y. Dissection and fine-mapping of two QTL for grain size linked in a 460-kb region on chromosome 1 of rice. Rice 2018, 11, 44. [CrossRef]

37. Wang, Z.; Chen, J.Y.; Zhu, Y.J.; Fan, Y.Y.; Zhuang, J.Y. Validation of $q$ GS10, a quantitative trait locus for grain size on the long arm of chromosome 10 in rice (Oryza sativa L.). J. Integr. Agric. 2017, 16, 16-26. [CrossRef]

38. Zhu, Y.J.; Huang, D.R.; Fan, Y.Y.; Zhang, Z.H.; Ying, J.Z.; Zhuang, J.Y. Detection of QTLs for yield heterosis in rice using a RIL population and its testcross population. Int. J. Genomics 2016, 2016, 2587823. [CrossRef]

39. Mei, D.Y.; Zhu, Y.J.; Yu, Y.H.; Fan, Y.Y.; Huang, D.R.; Zhuang, J.Y. Quantitative trait loci for grain chalkiness and endosperm transparency detected in three recombinant inbred line populations of indica rice. J. Integr. Agric. 2013, 12, 1-11. [CrossRef]

40. Zhu, Y.J.; Zhang, Z.H.; Chen, J.Y.; Fan, Y.Y.; Mou, T.M.; Tang, S.Q.; Zhuang, J.Y. Fine mapping of $q$ TGW10-20.8, a QTL having important contribution to grain weight variation in rice. Crop J. 2019, 1, 587-597. [CrossRef]

41. Zhang, H.; Zhu, Y.J.; Fan, Y.Y.; Huang, T.X.; Zhang, J.F.; Xie, H.A.; Zhuang, J.Y. Identification and verification of quantitative trait loci for eating and cooking quality of rice (Oryza sativa). Plant Breed. 2019, 138, 568-576. [CrossRef]

42. Sun, Z.C.; Zhu, Y.J.; Chen, J.Y.; Zhang, H.; Zhang, Z.H.; Niu, X.J.; Fan, Y.Y.; Zhuang, J.Y. Minor-effect QTL for heading date detected in crosses between indica rice cultivar Teqing and near isogenic lines of IR24. Crop J. 2018, 6, 291-298. [CrossRef]

43. Zheng, K.; Huang, N.; Bennett, J.; Khush, G.S. PCR-based marker-assisted selection in rice breeding. In IRRI Discussion Paper Series No.12; International Rice Research Institute: Los Banos, Philippines, 1995.

44. Chen, X.; Temnykh, S.; Xu, Y.; Cho, Y.G.; McCouch, S.R. Development of a microsatellite framework map providing genome-wide coverage in rice (Oryza sativa L.). Theor. Appl. Genet. 1997, 95, 553-567. [CrossRef]

45. Lander, E.S.; Green, P.; Abrahamson, J.; Barlow, A.; Daly, M.J.; Lincoln, S.E.; Newberg, L.A. MAPMARKER: An interactive computer package for constructing primary genetic linkage maps of experimental and natural populations. Genomics 1987, 1, 174-181. [CrossRef]

46. Meng, L.; Li, H.; Zhang, L.; Wang, J. QTL IciMapping: Integrated software for genetic linkage map construction and quantitative trait locus mapping in biparental populations. Crop J. 2015, 3, 269-283. [CrossRef]

47. McCouch, S.R.; CGSNL (Committee on Gene Symbolization, Nomenclature and Linkage, Rice Genetics Cooperative). Gene nomenclature system for rice. Rice 2008, 1, 72-84. [CrossRef] 
48. Wang, S.C.; Basten, J.; Zeng, Z.B. Windows QTL Cartographer 2.5, Department of Statistics; North Carolina State University: Raleigh, NC, USA, 2012.

49. Tian, F.; Li, D.J.; Fu, Q.; Zhu, Z.F.; Fu, Y.C.; Wang, X.K.; Sun, C.Q. Construction of introgression lines carrying wild rice (Oryza rufipogon Griff.) segments in cultivated rice (Oryza sativa L.) background and characterization of introgressed segments associated with yield-related traits. Theor. Appl. Genet. 2006, 112, 570-580. [CrossRef]

50. Thomson, M.J.; Tai, T.H.; McClung, A.M.; Lai, X.H.; Hinga, M.E.; Lobos, K.B.; Xu, Y.; Martinez, C.P.; McCouch, S.R. Mapping quantitative trait loci for yield, yield components and morphological traits in an advanced backcross population between Oryza rufipogon and the Oryza sativa cultivar Jefferson. Theor. Appl. Genet. 2003, 107, 479-493. [CrossRef]

51. Zhu, A.; Sun, Z.; Zhu, Y.; Zhang, H.; Niu, X.; Fan, Y.; Zhang, Z.; Zhuang, J. Identification of QTL for grain weight and grain shape using populations derived from residual heterozygous lines of indica rice. Chin. J. Rice Sci. 2019, 33, 144-151.

52. Wu, W.; Zheng, X.M.; Luo, G.; Zhong, Z.; Gao, H.; Chen, L.; Wu, C.; Wang, H.J.; Wang, Q.; Zhou, K.; et al. Association of functional nucleotide polymorphisms at $D T H 2$ with the northward expansion of rice cultivation in Asia. Proc. Natl. Acad. Sci. USA 2013, 110, 2775-2780. [CrossRef]

53. Hu, J.; Wang, Y.; Fang, Y.; Zeng, L.; Xu, J.; Yu, H.; Shi, Z.; Pan, J.; Zhang, D.; Kang, S.; et al. A rare allele of GS2 enhances grain size and grain yield in rice. Mol. Plant 2015, 8, 1455-1465. [CrossRef] [PubMed]

54. Qi, P.; Liu, Y.S.; Song, X.J.; Shen, J.B.; Huang, W.; Shan, J.X.; Zhu, M.Z.; Jiang, L.W.; Gao, J.P.; Lin, H.X. The novel quantitative trait locus GL3.1 controls rice grain size and yield by regulating Cyclin-T1;3. Cell Res. 2012, 22, 1666-1680. [CrossRef] [PubMed]

55. Fan, Y.Y.; Chen, C.; Wu, J.R.; Cheng, S.H.; Zhuang, J.Y. Quantitative trait loci for yield traits located between $H d 3 a$ and Hd1 on short arm of chromosome 6 in rice. Rice Sci. 2011, 18, 257-264. [CrossRef]

(C) 2020 by the authors. Licensee MDPI, Basel, Switzerland. This article is an open access article distributed under the terms and conditions of the Creative Commons Attribution (CC BY) license (http://creativecommons.org/licenses/by/4.0/). 